\title{
6 \\ Rotation of melting ice disks due to melt fluid flow
}

\author{
S. Dorbolo, N. Adami, C. Dubois, H. Caps, N. Vandewalle, and B. Darbois-Texier \\ CESAM-GRASP, Département de Physique B5, Université de Liège, B-4000 Liège, Belgium
}

(Received 23 October 2015; published 14 March 2016)

\begin{abstract}
We report experiments concerning the melting of ice disks ( $85 \mathrm{~mm}$ in diameter and $14 \mathrm{~mm}$ in height) at the surface of a thermalized water bath. During the melting, the ice disks undergo translational and rotational motions. In particular, the disks rotate. The rotation speed has been found to increase with the bath temperature. We investigated the flow under the bottom face of the ice disks by a particle image velocimetry technique. We find that the flow goes downwards and also rotates horizontally, so that a vertical vortex is generated under the ice disk. The proposed mechanism is the following. In the vicinity of the bottom face of the disk, the water eventually reaches the temperature of $4{ }^{\circ} \mathrm{C}$ for which the water density is maximum. The $4{ }^{\circ} \mathrm{C}$ water sinks and generates a downwards plume. The observed vertical vorticity results from the flow in the plume. Finally, by viscous entrainment, the horizontal rotation of the flow induces the solid rotation of the ice block. This mechanism seems generic: any vertical flow that generates a vortex will induce the rotation of a floating object.
\end{abstract}

DOI: 10.1103/PhysRevE.93.033112

\section{INTRODUCTION}

Large floating ice blocks have been observed on frozen rivers [1-4]. This pretty rare phenomenon is shown during winter news broadcasts on TV as the disks surprisingly rotate [5]. In a paper of 1997, Nordell et al. reported several events of rotating ice disks [1]. The diameter $D$ of the disks has been observed to range over two orders of magnitude from 1 to $100 \mathrm{~m}$. On the other hand, the rotation speeds do not seem to depend on the size of the disk. They observed that angular rotation speed $\omega$ is of the order of $1^{\circ} / \mathrm{s}$. The mechanism of the disk formation is still to be discovered in detail. Nowadays, two hypotheses are discussed: either the disk is created by the aggregation of frazil ice in a vortex generated by the river or an ice block is formed before being rounded [1]. As the natural ice disks float on a river, the rotation is probably driven by the flow, the disk working like a water turbine.

In the present paper, we investigate the role of the flow: Is it possible that only the melting of the ice can generate a motion of the ice block? For this purpose, we studied centimetric ice disks floating at the surface of a pool of water maintained at a given temperature. We discovered that the disks start rotating spontaneously while melting. In particular, we focused on the disk rotation engendered by the flow of the melted ice. Indeed, we found that, while melting, the ice disk generates a vertical vortex under the immersed surface of the disk. This vortex is sufficient to trigger the rotation of the ice disk.

\section{EXPERIMENTAL SETUP}

The water pool consisted of a large stainless steel circular vessel (300 $\mathrm{mm}$ in diameter and $150 \mathrm{~mm}$ in depth). The water pool was plunged in an intermediate bath whose temperature was regulated by flowing thermalized water through a copper serpentine (see Fig. 1). The thermalized water was produced by a flow thermostat (Julabo F12-ED). Finally, two caps were placed: one at the surface of the intermediate bath (where a pump mixed the liquid for homogenization purposes) and one over the whole experiment in order to avoid any perturbation by the air environment.

Tap water was poured into a circular Petri dish $(85 \mathrm{~mm}$ in diameter $D$ and $14 \mathrm{~mm}$ in height $e$ ) before freezing. In order to obtain clear ice (without bubbles), the freezing front started at the bottom of the Petri dish. Indeed as the water started freezing from the bottom of the Petri dish upwards, bubbles and dust were pushed towards the surface of the water. Therefore, the Petri dish was embedded in polystyrene except the bottom face. The polystyrene frame was placed in a freezer $\left(-32^{\circ} \mathrm{C}\right)$.

A dark ellipse (about $50 \mathrm{~mm}$ long and $20 \mathrm{~mm}$ wide ) was put on the floating ice disk. By image analysis, the position of the center of the ellipse and its angular position were measured and allowed us to determine the motion of the ice disk during the melting. The images were captured from a webcam and treated in real time using a Python program and the OpenCV library.

While melting, we observed that the ice disks moved at the surface of the bath. Sometimes they were rotating similarly to the spinning ice disks observed in rivers. Sometimes they were rotating and translating at the surface of the bath. The ellipse contours have been superimposed to deduce the motion of the disks ( $1 \mathrm{~s}$ separates each successive position of the ellipse). Two typical trajectories of free floating ice disks are presented in Fig. 2 on the left. One can see from left to right: a combination of two rotations and the combination of a translation and a rotation motion. In order to increase the reproducibility, we suppressed the translational degrees of freedom by constraining the ice disk along a vertical axis crossing the disk at its center. A small nickel bead $(2 \mathrm{~mm}$ of diameter) was embedded at the bottom center of the ice disks before freezing. A fixed magnet was placed above the surface of the bath. This magnet attracted the nickel bead and consequently the ice disk. A typical trajectory of a constrained ice disk is shown on the right of Fig. 2. Note that nickel was chosen because (i) nickel does not oxidize and (ii) nickel is ferromagnetic with a small remanent field at $273 \mathrm{~K}$. Indeed, if a magnet was placed in the ice disk instead of the $\mathrm{Ni}$ bead, a torque on the disk may disturb the disk melting and consequently the disk motion.

\section{OBSERVATIONS AND RESULTS}

In Fig. 3, the rotation speed $\omega(\% / \mathrm{s})$ is plotted as a function of the temperature of the pool. We observe that, even constrained 


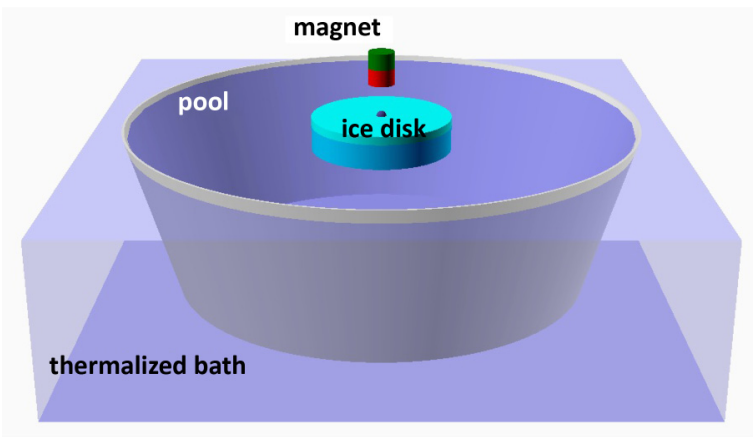

FIG. 1. The ice disks were placed at the surface of the water pool. The temperature of the water pool was controlled by an intermediated bath of thermalization. The ice disk was either free or magnetically constrained along a vertical axis passing through the center of the disk. In the latter case, a nickel bead was embedded in the ice disk. The serpentine is not represented.

about the vertical axis, the data scatter quite a bit. The data were binned by $5{ }^{\circ} \mathrm{C}$ boxes and averaged (big bullets in Fig. 3 ). A clear increase of the rotation speed is observed when the temperature of the pool is increased. The rotation starts to be efficient for a temperature larger than $5{ }^{\circ} \mathrm{C}$. This indicates that the origin of the rotation is related to the so-called density anomaly of water, i.e., the density of the water is maximal at a temperature of $4{ }^{\circ} \mathrm{C}$.

In order to visualize the motion of the fluid below the ice disks, we colorized the water used to make the disks. This simple experiment allowed observing the formation of a vortex below the ice disk. The radius of the vortex was typically half that of the disk.

Quantitative results were obtained by using particle image velocimetry (PIV) technique from DantecDynamics. Fluorescent particles were inserted in the liquid bath which was poured into a transparent tank. For this part, the bath temperature was $20^{\circ} \mathrm{C}$. A green LASER was used for the excitation and a Phantom camera recorded the motion of the particles. The ice disk was constrained to rotate about the vertical axis defined by the magnet and the Ni bead contained in the ice disk. The PIV investigations were performed along two perpendicular planes: (A) one plane $(x-z)$ was taken perpendicular to the bath surface intercepting the axis of rotation of the ice disk and (B) one plane $(x-y)$ was taken parallel to the bath surface
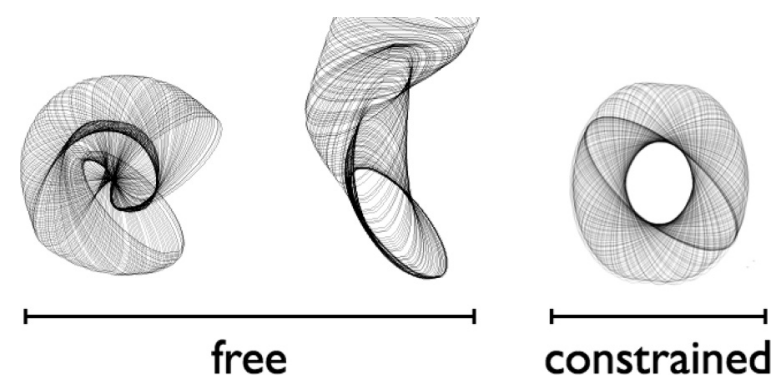

FIG. 2. Left and center: Superposition of the ellipse contours over time showing the typical trajectories of the motion of the ice disks while free floating. Right: Trajectory of an ice disk when the disk was constrained rotating around its center. The large axis of the ellipse is $50 \mathrm{~mm}$ and the temperature of the thermalized pool is $20^{\circ} \mathrm{C}$.

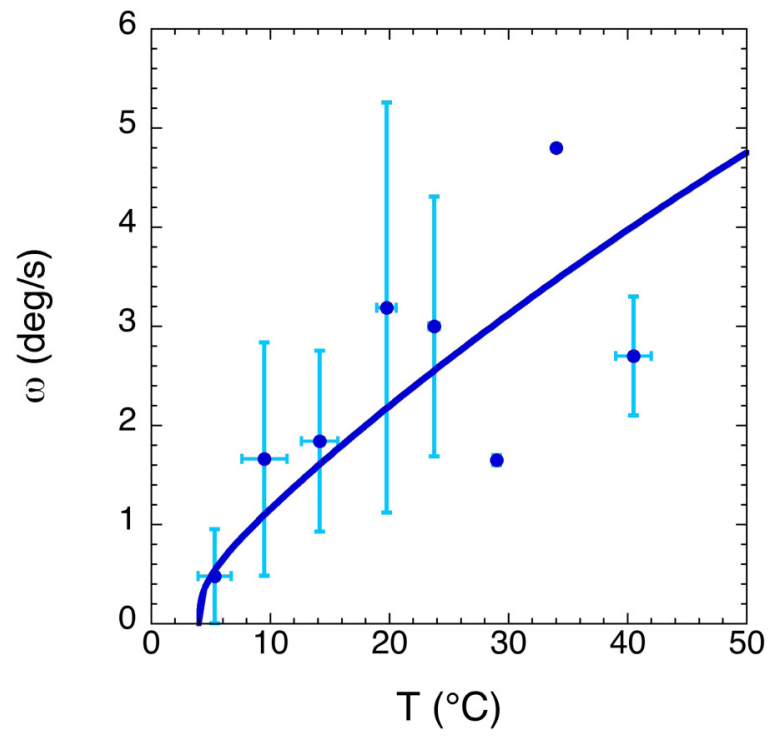

FIG. 3. Rotation speed $\omega$ of the constrained ice disk expressed in degrees per second as a function of the pool temperature $T$. The bullets are obtained by averaging the data over bins of $5^{\circ} \mathrm{C}$. The solid line is a fit from Eq. (12). The lack of error bars for two of the points is due to the way the data were binned.

about $5 \mathrm{~mm}$ below the bottom surface of the ice disk (see Fig. 4).

In Fig. 4, the velocity fields are presented for both concerned planes (parallel $x-y$ and perpendicular $x-z$ ). First, concerning the vertical plane $x-z$, we observe that the fluid moves towards the center of the ice disk located around $x=0 \mathrm{~mm}$. The maximum speed observed at $15 \mathrm{~mm}$ below the bottom face of the ice disk $(z=0 \mathrm{~mm})$ is about $10 \mathrm{~mm} / \mathrm{s}$ downwards. The analysis of the horizontal plane $x-y$ also indicates maximum speed of the same order of magnitude. Moreover, a horizontal rotation of the flow is observed below the ice disk, in the present case around a center located at $x=-10 \mathrm{~mm}$ and $y=10 \mathrm{~mm}$. Regarding these observations, we conclude that a downwards flow is generated below the ice which creates a vertical vortex. The situation is similar to the generation of a vortex during the emptying of a bathtub by the plughole [6].

The proposed mechanism is the following. The ice disk melts and decreases locally the water temperature close to the bottom surface of the disk. Eventually, the water reaches locally the temperature of $4{ }^{\circ} \mathrm{C}$. At this temperature the density of the water is the highest and the fluid sinks towards the bottom of the pool and generates a plume. The plume is similar to the thermal plume observed in soap films $[7,8]$. The plume induces the horizontal rotation of the fluid, i.e., a vertical vortex close to the bottom face of the ice disk. We assess that the vortex puts the ice disk in rotation by a viscous entrainment process. Balancing the entrainment force and the friction force results in a constant speed of rotation. Note that this mechanism is also supported by the fact that when the level of the water in the pool is low $(3 \mathrm{~cm})$, the ice disk does not rotate.

The thermal convection dominates as confirmed by the evaluation of the Rayleigh number

$$
\mathrm{Ra}=\frac{g \alpha \rho_{0} L^{3} \Delta T}{\eta D}
$$


(a)

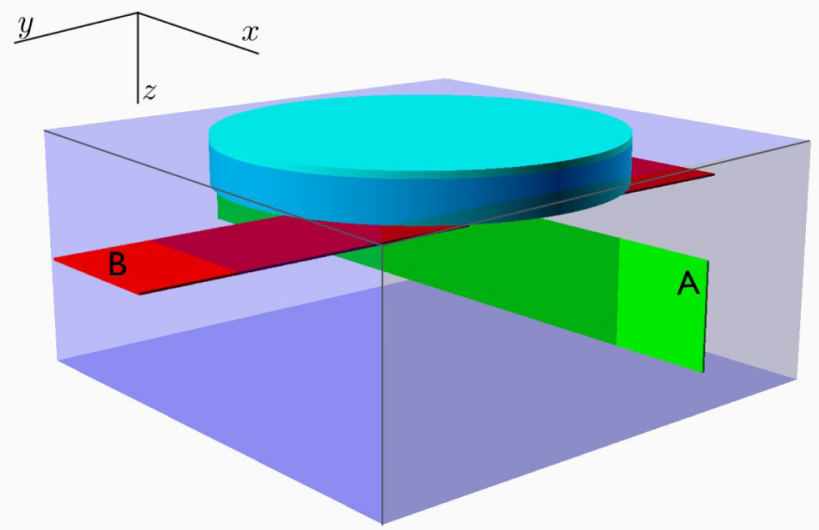

(b)

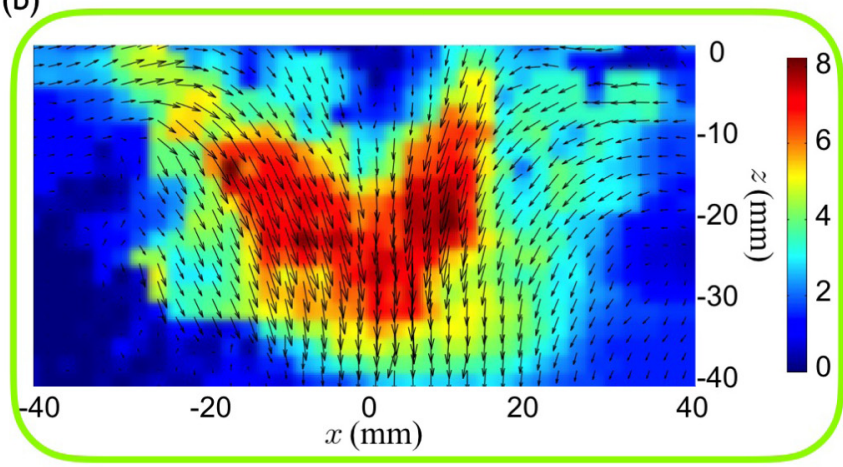

(c)

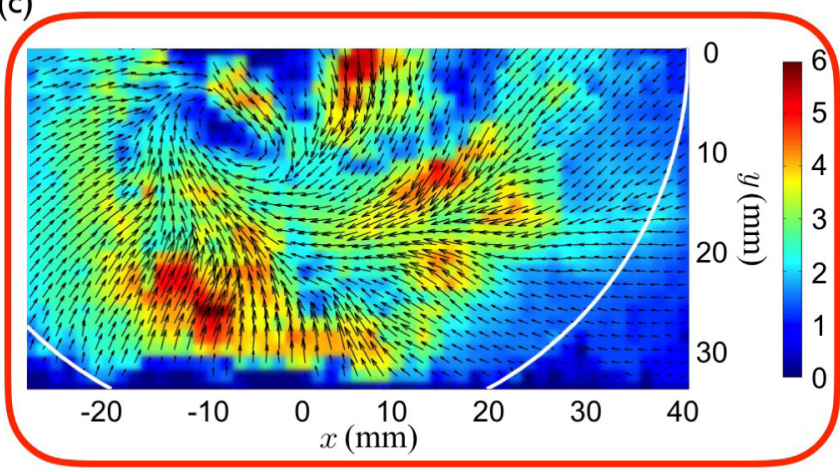

FIG. 4. Particle imaging velocimetry measurements at $T=$ $20^{\circ} \mathrm{C}$. (a) Sketch of the orientation of the planes along which the PIV measurements were performed, namely (A) one perpendicular $(x-z)$ and (B) one parallel $(x-y)$ to the bath surface (see text). The results for the perpendicular and the parallel planes are presented in (b) and (c) respectively. The color scales indicates the speed in $\mathrm{mm} / \mathrm{s}$.

where $\alpha, D, \eta$, and $\rho_{0}$ are the thermal expansion coefficient $\left(207 \times 10^{-6} \mathrm{~K}^{-1}\right.$ at $\left.20^{\circ} \mathrm{C}\right)$, the thermal diffusivity $(1.5 \times$ $\left.10^{-7} \mathrm{~m}^{2} \mathrm{~s}^{-1}\right)$, the kinetic viscosity $\left(10^{-3} \mathrm{~Pa} \mathrm{~s}\right)$, and the density $\left(1000 \mathrm{~kg} \mathrm{~m}^{-3}\right)$ of the water, respectively, $g$ is the gravity acceleration, and $L(=R)$ is the characteristic length of the system. One finds $\mathrm{Ra} \approx 20 \times 10^{6}$ when the bath is at a temperature of $20^{\circ} \mathrm{C}$. To model the plume, we follow Ref. [9]. The buoyancy flux $F$ is defined as

$$
F=g \alpha T \pi R_{p}^{2}(z) v_{z}(z),
$$

where $g \alpha T$ is the reduced gravity due to buoyancy and $\pi R_{p}^{2}(z) v_{z}(z)$ is the volumetric flow; $v_{z}$ is the average vertical

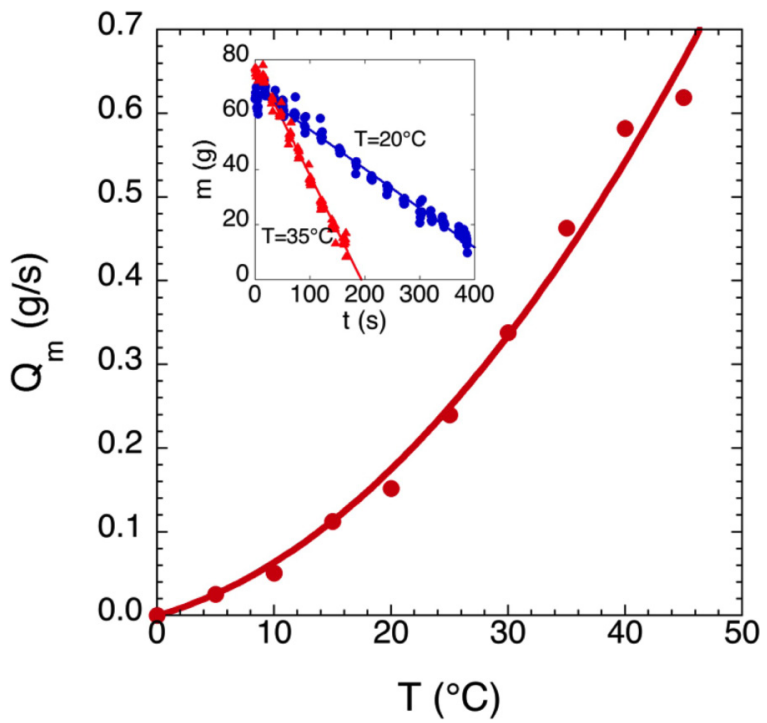

FIG. 5. Mass flux of the ice disk as a function of the temperature. The line is the fit from Eq. (6). The inset presents the mass decrease of two ice disks as a function of time for two different temperature, i.e. $T=20^{\circ} \mathrm{C}$ (blue bullets) and $T=35^{\circ} \mathrm{C}$ (red triangles).

speed and $R_{p}(z)$ is the radius of the cross section of the plume. One obtains the buoyancy flux as a function of the thermal flux $Q_{T}$ by noting that $Q_{T}=\rho_{0} C_{p} T \pi R_{p}^{2} v_{z}$ where $\rho_{0}$ and $C_{p}$ are the density and the specific heat of the water, namely

$$
F=\frac{\alpha g}{\rho_{0} C_{p}} Q_{T}
$$

Equation (3) allows us to obtain a simple expression for $v_{z}$ as a function of the temperature and the position in the bath. Taking into account the heat, the mass, and the momentum budget on a thin slice, Cushman-Roisin [9] deduces an expression for the velocity $v_{z}$ of the fluid at the center of the plume,

$$
v_{z} \propto\left(\frac{F}{z}\right)^{1 / 3},
$$

where $z$ is the vertical position with $z=0$ corresponding to the location of the ice disk bottom face. The thermal flux $Q_{T}$ can be evaluated by determining the mass flux $Q_{m}$ of the ice disk if we consider that the heat is used to change the ice into water plus the heat necessary to heat the ice from -30 to $0{ }^{\circ} \mathrm{C}$ $\left(\Delta T=30^{\circ} \mathrm{C}\right)$,

$$
Q_{T}=\left(\mathcal{L}+C_{\text {ice }} \Delta T\right) Q_{m},
$$

where $\mathcal{L}$ and $C_{\text {ice }}$ are the latent heat of fusion and the specific heat of ice, respectively. The mass flux can be easily estimated. The procedure consisted of weighting the ice disk as a function of time by briefly pulling the disk out of the pool using a string attached to a force sensor. In the inset of Fig. 5, the mass $m$ of the ice disk is presented as a function of time when the pool is at $20^{\circ} \mathrm{C}$ (red triangles) and at $35^{\circ} \mathrm{C}$ (blue circles). The evolutions of $m(t)$ were fitted by a linear trend $Q_{m} t$ for different temperatures of the pool. The angular coefficients $Q_{m}$ are reported as a function of the pool temperature in Fig. 5. In 


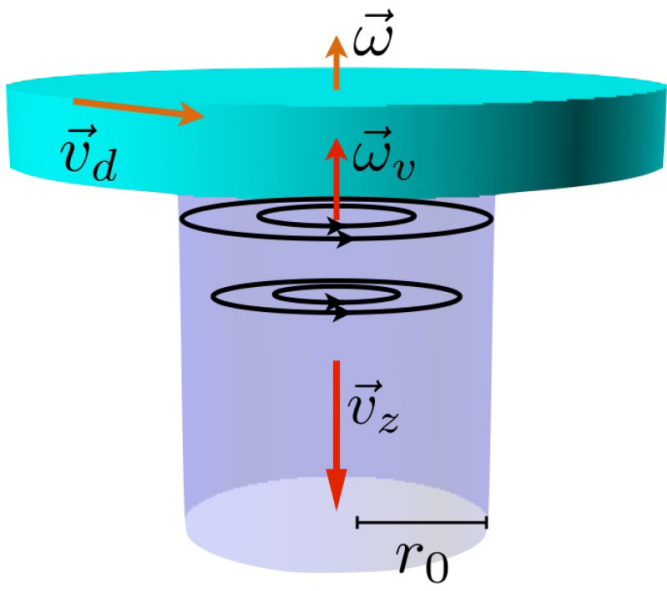

FIG. 6. Sketch of the side view of the melting ice disk. A vertical plume directed downward leads to the vertical motion of the liquid with a vertical speed $v_{z}$. The vertical motion of the fluid generates a vertical vortex with an angular velocity $\omega_{v}$ which entrains the rotation of the ice disk with an angular velocity $\omega$.

the considered range of temperature, one finds that

$$
Q_{m}=3.9 \times 10^{-6} T+240 \times 10^{-9} T^{2} .
$$

With the assumption that the vertical speed is due to the water anomaly we find by combining Eqs. (4)-(6) that

$$
v_{z} \propto\left(\frac{\alpha(T) g}{z \rho_{0} C_{p}} Q_{m}\left(\mathcal{L}+C_{\text {ice }} \Delta T\right)\right)^{1 / 3}
$$

taking $\alpha(T)=14 \times 10^{-6}(T-4)$ (in the range $4-50{ }^{\circ} \mathrm{C}$ ) [10], $\rho_{0}=1000 \mathrm{~kg} / \mathrm{m}^{3}, C_{p}=4202 \mathrm{~J} \mathrm{~kg}^{-1} \mathrm{~K}^{-1}, \quad C_{\text {ice }}=$ $2060 \mathrm{~J} \mathrm{~kg}^{-1} \mathrm{~K}^{-1}, \Delta T=30^{\circ} \mathrm{C}$, and $z=15 \mathrm{~mm}$ as in the PIV measurement, one finds $v_{z} \approx 12 \mathrm{~mm} / \mathrm{s}$. This value is of the same order of magnitude as the speed found by the PIV measurements even if the effect of the thermal gradient is not taken into account. Note that the dependence of the vertical speed with the temperature comes from the temperature dependence of the thermal expansion $\alpha$.

The relation between the vertical flow and the vertical vortex velocity is rather complex (see for example [11]). In the present case, the vertical flow is driven by natural convection. Phenomenologically speaking, one can assess that (i) the vertical speed $v_{z}$ of the plume is of the same order of magnitude as the horizontal maximum speed of the fluid and (ii) the vortex radius $r_{0}$ (the same as the plume cross section radius $R_{p}$ ) is half the radius $R$ of the ice disk (see Fig. 6).

We postulate that the horizontal fluid rotation exerts a viscous entrainment on the ice disk which starts rotating. Providing that the viscous entrainment is produced on a boundary layer of a thickness $\delta$, the driving force $f$ is given by

$$
f \simeq \int_{0}^{r_{0}} \int_{0}^{2 \pi} \eta \frac{\left(\omega_{v}-\omega\right) r}{\delta} r d \theta d r=2 \pi r_{0}^{3} \frac{\eta\left(\omega_{v}-\omega\right)}{3 \delta},
$$

where $\eta$ is the dynamic viscosity of the water. On the other hand, the friction of the disk with the pool limits the rotation. This friction occurs along the lateral surface of the disk and on the remaining surface on the bottom side of the disk. Taking

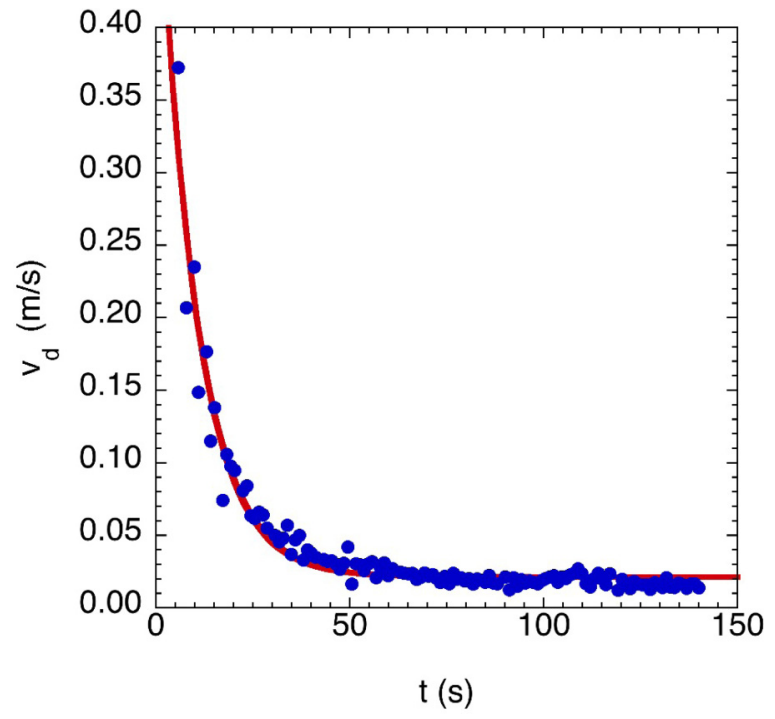

FIG. 7. Tangential linear speed $v_{d}$ of the disk as a function of time when the disk was initially rotated by hand. The plain line is a fit by an exponential decay.

the same argument as for the entrainment, the friction force due to viscosity on a layer of thickness $\delta$ reads

$$
\begin{aligned}
f_{d} & =\int_{0}^{2 \pi} \eta \frac{\omega R}{\delta} e R d \theta+\int_{r_{0}}^{R} \int_{0}^{2 \pi} \eta \frac{\omega r}{\delta} r d \theta d r, \\
f_{d} & =2 \pi \eta \frac{\omega}{\delta}\left(e R^{2}+\frac{1}{3}\left(R^{3}-r_{0}^{3}\right)\right),
\end{aligned}
$$

where $e$ is the thickness of the ice disk. As the rotation speed of the disk is constant, the forces balance and we obtain a relation for the coupling $\gamma$ :

$$
\gamma=\frac{\omega}{\omega_{v}}=\frac{r_{0}^{3}}{R^{3}} \frac{1}{1+3 e / R} .
$$

Applied to the present case, one finds that $\frac{\omega}{\omega_{v}} \approx 0.06$ with $e=14 \mathrm{~mm}$. The layer thickness $\delta$ can be evaluated as follows. Ice disks were rotated by hand and released. The rotation motion was recorded and consequently, the linear speed $v_{d}=$ $\omega R$ was computed as a function of time. In Fig. 7, the tangential speed of the disk is reported as a function of time. The data were fitted by an exponential decay. We find a relaxation rate $\kappa \approx 0.1 \mathrm{~s}^{-1}$. This behavior indicates that the tangential speed may be modelled by a differential equation $m \dot{v}_{d}=-m \kappa v_{d}$ where $m$ is the mass of the ice disk. The decrease of the speed comes from the friction $f_{h d}$ of the disk (lateral surface and bottom side) with liquid. The calculation is similar to Eq. (9). The integral is performed from 0 to $R$ instead of between $r_{0}$ and $R$. One obtains

$$
f_{h d}=\frac{2 \pi \eta \omega R^{3}}{\delta}\left(\frac{e}{R}+\frac{1}{3}\right)=m \kappa \omega R .
$$

Consequently, the thickness $\delta$ of the boundary layer can be estimated, i.e., $\delta \approx 233 \mu \mathrm{m}$.

Finally, the rotation speed $\omega$ can be deduced from Eqs. (7) and (10). In so doing, one finds that

$$
\omega \sim \gamma(F / z)^{1 / 3}
$$


The blue curve in Fig. 3 is the fit to the data using this scaling where $z=15 \mathrm{~mm}$. The agreement is fairly good as only the aspect ratio of the vortex embedded in the parameter $A$ (see Ref. [9]) has been left free. Note also that the viscosity of the water does not intervene in the final description of the rotation speed Eq. (10). Indeed, as the driving force $f$ and the friction force $f_{d}$ have the same physical origin, the role of the viscosity cancels.

\section{CONCLUSION}

The spontaneous rotation of a melting ice disk has been studied at the laboratory scale. The rotation speed increases with the bath temperature. The mechanism is related to the density anomaly of the water at $4{ }^{\circ} \mathrm{C}$. Indeed, the ice disk cooled the water of the pool. As the water reaches the temperature of $4{ }^{\circ} \mathrm{C}$, the water sinks generating a downward plume and a subsequent downward flow. This flow triggers a vertical vortex that puts in rotation the ice disk by a viscous entrainment process. This allows us to understand why such a process cannot be observed in lakes because the water deep in the lake is at $4^{\circ} \mathrm{C}$ while the water in between the ice and the bottom is between 0 and $4{ }^{\circ} \mathrm{C}$. The thermal plume cannot form. In rivers, the situation is different as the flow is supposed to be sufficient to generate the rotation. One also understands why a melting iceberg does not rotate as the melting results in diluting salted seawater. Finally, we notice that this mechanism is generic. For example, a caramel disk has been attached to a floating circular boat. The melting of the caramel has generated the rotation of the boat.

\section{ACKNOWLEDGMENTS}

S.D. acknowledges support as a FNRS Senior Research Associate. N.V. thanks the Action de Recherche Concertée (FWB) ARC-Quandrops project. H.C. and C.D. thanks the Action de Recherche Concertée (FWB) ARC-Supercool project. This research has been also funded by the IAP 7/38 MicroMAST initiated by the Belgian Science Policy Office (BELSPO).
[1] B. Nordell and G. Westerstrom, Weather 52, 17 (1997).

[2] E. Hvolboll, EOS 64, cover image (1983).

[3] J. M. Bates, Sci. Am. 72(6), 85 (1895).

[4] T. Carstens, Houille Blanche 7, 642 (1971).

[5] Spinning ice disk phenomenon seen in British river for first time, The Telegraph, Jan. 13th (2009).

[6] A. Andersen, T. Bohr, B. Stenum, J. J. Rsmussen, and B. Lautrup, Phys. Rev. Lett. 91, 104502 (2003).
[7] N. Adami, S. Dorbolo, and H. Caps, Phys. Rev. E 84, 046316 (2011).

[8] B. Martin and X. L. Wu, Phys. Rev. Lett. 80, 1892 (1998).

[9] B. Cushman-Roisin, Environmental Fluid Mechanics (Thayer School of Engineering, Hanover, 2015).

[10] Water-Thermal properties, The Engineering Toolbox.

[11] W. S. Lewellen, J. Fluid Mech. 14, 420 (1962). 